\title{
Matter and Gravity in Warped Extradimensional Models: Reinterpreting Randall-Sundrum
}

\author{
Katherine Benson* \\ Emory University Physics Department \\ Atlanta, Georgia 30322-2430, USA \\ E-mail: benson@physics.emory.edu
}

\begin{abstract}
Warped extra dimension claims remarkable success: solving the hierarchy problem; explaining hierarchies in particle phenomenology; yielding standard cosmology, plus interesting nonstandard scenarios. Yet it has marked shortcomings: we over-rely on a single toy model, Randall-Sundrum; we treat matter and gravity in an ad hoc, asymmetric way; and we conceptualize integrated 4D effective field theory inconsistently. I here construct sounder 4D effective field theories for matter and gravity in warped extra dimension - whether Randall-Sundrum or higher codimension. I track both Planck and particle scales through brane formation, beginning with fully extradimensional matter and gravity, at unified scale, in gravitationally warped backgrounds with bulk electroweak symmetry-breaking. This validates hierarchy solution, as warp generically drives 4D effective Planck and particle scales apart. It evades classic obstacles to warped confinement of matter: colocalizing particles and assuring effective charge universality. Diverse particles do fail to colocalize, generically and in discussed models; however, aggregate 4D effective field theory still holds, since hierarchy solution fixes unresolvably small extradimensional radius. Electromagnetic charge universality emerges generically, and weak charge universality in the RandallSundrum case.
\end{abstract}

28th Johns Hopkins Workshop on Current Problems in Particle Theory

June 5-8, 2004

Johns Hopkins University Homewood campus - Bloomberg Center for Physics and Astronomy, Baltimore, Maryland

*Speaker. 


\section{Introduction: The origins of warped extra dimension}

In the past century, theorists' attempts to reconcile gravity and particle interactions have involved a recurrent theme, of extra spatial dimensions in our universe. This notion arose in the 1920s, when Kaluza and Klein tried to model electromagnetism as gravitational dynamics of a compactified extra dimension. [1], 2] It reappeared in supergravity, string and M theory: unified treatments of quantum gravity and particle interactions, consistent only in higher dimension. Early theorists viewed the required extra dimensions as unresolvably small, with no measurable impact on observed 4D physics. Brane solutions to string theory - defects confining matter, but not gravity, through stringy effects [3, 4] - changed that view. With matter confined to 4D branes, dilution of gravity in large extra dimensions could explain its hierarchical weakness, without violating 4D observation. [5] This dilution scales with volume, for flat extra dimensions. However, as defects, branes typically carry tension, gravitationally warping the extra dimensions. In 5D toy models, Randall and Sundrum showed this gravitational warp can accomplish two things: binding massless gravitons, to induce 4D effective gravity on a brane; [6] and generating hierarchy between apparent Planck and particle scales, on a (different) brane. [7] Thus warped extra dimension emerged in 1999, as a new reconciler of gravity and particle interactions, rendering both effectively 4D and inducing their hierarchy from a single extradimensional scale.

The Randall-Sundrum models established a paradigm for warped extra dimension, wherein the apparent dimension of our universe dynamically shifts to 4 , when branes form, simultaneously binding matter and gravity, and generating their hierarchy. This paradigm sparked extensive research: both Randall-Sundrum papers amassed more than 2000 citations, and ranked in the top 10 for high energy physics citations each year since 2000, according to the SLAC SPIRES database. This has two reasons. First, the Randall-Sundrum models are toy models par excellence: simple, calculable, with deep connections to fundamental paradigms like braneworld physics, AdS-CFT issues, and the Horava-Witten vacuum in string theory. Second, their application generated phenomenal success, in both phenomenology and cosmology. Within cosmology, for example, they reproduce standard Friedmann-Robertson-Walker universe evolution for late times, in one-brane [8, 9, 10, 11] and two-brane variants [12, 13, 14,; alter inflationary physics in acceptable and potentially interesting ways [15, 16, 17]; or seed late-time quintessence. [18, 14] These successes can be transformative for particle cosmology.

I note that such success is not unparalleled: flat models of extra dimension generate a comparable literature of phenomenological and cosmological achievements, particularly in two models, the original large extra dimensions proposal [5] and a subsequent "infinite volume" extra dimensions proposal. [19] However, warped extra dimension has more natural appeal. For large, "infinite volume," and warped extradimensional models are all intrinsically braneworld models, deriving their viability from the dynamical confinement of ordinary matter on branes. Yet branes typically carry nonzero tension, which itself generically warps spacetime.

\section{Critique: Shortcomings in the paradigm}

The success of warped extra dimension, in solving the hierarchy problem; explaining hierarchies within particle physics; replicating 4D gravity and standard big bang cosmology; and seed- 
ing inflationary and quintessential dynamics is spectacular. However, serious shortcomings persist, motivating the work I discuss today. These shortcomings have two origins: first, over-reliance on the Randall-Sundrum model to represent the warped extradimensional paradigm; and second, ad hoc and asymmetric treatment of matter and gravity effective theories in warped extradimensional backgrounds.

The first shortcoming stems simply from overreach. For all their promise, phenomenological and cosmological results nearly all arise in one narrow context: Randall-Sundrum's toy models, with simple extensions (bulk scalar fields - themselves necessary to stabilize Randall-Sundrum I [20]; bulk gauge fields [21, 22]; adding cosmological energy density to Randall-Sundrum backgrounds [18], and to detuned Randall-Sundrum backgrounds, with $\Lambda_{4} \neq 0$ [18]). Warped extra dimension arises generically from braneworld physics; the Randall-Sundrum models, however, need not. They model a well-motivated vacuum solution to M theory, the Horava-Witten vacuum, with branes at fixed points of a large orbifolded extra dimension. [23] Yet the Horava-Witten vacuum is one string vacuum, among a multitude of contenders. [24, 25, 26, 27, 28, 29, Moreover, the very role of string vacua may be limited by finite temperature effects. Brandenberger and Vafa address dimensional reduction of string theory from this finite temperature perspective, asking in thermodynamic equilibrium how dimensions grow. For toroidal compactifications they find a dimension-selecting mechanism, with persistent cycle windings obstructing expansion in all but a dimensionally reduced subspace. [30] Recent analysis suggests that often 4 dimensions grow very large, while 2 grow to intermediate scale. [31, 32] Such arguments motivate, among myriad possibilities, scenarios with 2 large extra dimensions, warping when 3-branes form. Thus a codimension 2 warped extradimensional model, like [33, 34, 35, 36, 37, 38, 39, 40, 41, 42, 43, might act as final stage, completing the chain of dynamical dimensional reduction from 6 to 4 , just as Randall-Sundrum completes dimensional reduction to 5 to 4 after formation of a HoravaWitten vacuum. It is the general paradigm of warped extra dimension - more broadly than the Randall-Sundrum model - which dimensionally reduces our observed universe, when 3-branes form, warp spacetime, and induce effectively 4D observable physics. This warped braneworld mechanism completes a dynamical pattern of dimensional reduction; one whose pathways, however, are many. Warped extra dimension thus deserves generic study, examining its implications for 4D effective physics, for novel phenomenology and cosmology, not through the limited lens of a single toy model, but with an eye toward capturing the generic, defining the landscape of structures and signatures inexorably associated with warped extra dimension, regardless of model.

Some progress toward this more generic perspective has occurred. A promising direction, that of extradimensional defect models, returns to the inspiration of branes as defect solutions required by duality. These construct branes explicitly as field theoretic, topological defects, formed by an uncharged matter condensate in the extra dimensions. The defect warps the metric, which then binds gravitons to a 3-brane at the defect's core. These various solutions retain the basic advantages of the Randall-Sundrum model: they confine gravity to a brane; produce a massless bound graviton mediating effective 4D gravity; cause only mild gravitational corrections via Kaluza-Klein modes; and solve the hierarchy problem. [33, 34, 36, 37, 38, 39, 40, 44, 45, 46, 47, 48, 43, They also diversify the warped extradimensional universe paradigm, suggesting a plethora of models with varying codimension for the warped extra dimensions. Friedmann equations and brane cosmology have been examined in codimension two, reproducing — for a single model, with thickened branes 
— standard Friedmann-Robertson-Walker limits. [49] These generalizations enable us to begin separating generic from model-dependent features of warped extra dimension.

However, these generalizations suffer from the second shortcoming of warped extradimensional physics, shared by the Randall-Sundrum model itself. This is an ad hoc and asymmetric treatment of gravity and matter 4D effective field theory. These warped extradimensional models all derive localization of massless gravitons on the brane, and the resulting shift in Planck scale, from a fundamental extradimensional, to an induced 4D effective, value. Most however assume, a priori, localization of ordinary matter on the brane. All - even the most ambitious - assume an effective scale for the 4D localized matter, equating it to the extradimensional Planck scale. Historically this arose for two reasons: first, string theoretic arguments compel confinement of ordinary particles to the brane, where strings end; while confining gravity requires a distinct, nonstringy mechanism. Offering this mechanism was itself the first success of warped extra dimension. Second, a more symmetric treatment, confining matter to branes via the same gravitational warp that confines gravitons, is an enterprise with a long history. Despite some success [50, 51, 52], longstanding problems remain: first, in confining gauge fields; and second, in confining matter while preserving 4D effective charge universality. [53] Thus most authors apply the mechanism of gravitationally warped confinement only to gravitons. Those who attempt a parallel gravitational confinement of matter confine it only as defect zero modes; that is, as massless particles. [54 55] This is an impressive achievement, in establishing field theory mechanisms to implement stringy matter confinement on branes. However, it abstains on a question central to hierarchy solution: establishing particle mass scales on the brane.

As I argue in [56], this asymmetric treatment of 4D effective Planck and particle scales is untenable. However it is conceptualized, it is the single event of brane formation which simultaneously reduces both matter and gravity to effective 4D theories. Our warped extradimensional paradigm derives a sometimes marked shift in gravity's effective scale during that localization; yet it assumes the particle scale stays fixed. Solving the hierarchy problem requires more: a closer, more consistent accounting, tracking both particle and Planck scales from the same starting point, through brane formation, when both scales evolve to their effective 4D values in the nontrivial context of brane-warped geometry. Such tracking is needed, not only to validate hierarchy solution, but also to validate brane cosmology and phenomenology. For all rely centrally on calibrated particle interactions on the brane; interactions whose parameters may shift nontrivially in brane-warped backgrounds.

This asymmetric approach is exacerbated in the Randall-Sundrum two-brane model, which employs an eclectic treatment of integrated 4D effective field theory for gravity. 4D effective gravity has support localized on a single brane; however Randall-Sundrum treat its $4 \mathrm{D}$ effective action as salient, and equal, everywhere in the bulk. Moreover, they assume this equal bulk action remains locally resolved: with precisely this local resolution - this recasting of an integrated bulk action in terms of local warp factors - responsible for the generation of distinct 4D Planck scales on different branes. This attribution of an integrated action fully and simultaneously to all distinguishable points, without regard to the action's support at each, is murky; more dubious is the pointwise resolution of an already integrated effective action. A more conventional treatment would regard the integrated 4D effective action for gravity as either 1) localized on the 4D brane of its support; or 2) a bulk effect, describing an aggregate consequence of an extra dimension itself 
too tiny to be resolved.

\section{Program: Constructing valid braneworld effective theories}

The work I discuss today addresses these shortcomings of warped extra dimension, as practiced. First, I broaden the universe of viable warped extradimensional models beyond the RandallSundrum example, establishing generic features and structures. Second, I ratchet upward the standard for viability. I deem warped extradimensional models viable if 1 ) they determine a single, well-defined, 4D effective field theory for matter and gravity, which fully and symmetrically encodes all consequences of extradimensional warp; and 2) they induce hierarchy of particle and Planck scales, within that valid 4D effective theory.

Fundamental to a consistent and symmetric treatment of matter and gravity is a computational strategy to peel back the veil: to extend the history of both particle and Planck scale from today's $4 \mathrm{D}$ values backward, through their dynamical confinement onto 4D branes, to an earlier unified extradimensional scale. Viewed in forward rather than reverse, this requires consistent tracking of particle and Planck scales forward: from a single unified starting point, through brane formation, when both scales evolve to effective 4D values in the brane-warped background.

The only calculable tool for such tracking is extradimensional field theory: assuming particles have a definite, field theoretic description not only after they are confined to the 3-brane, but before, in an extradimensional universe. Such extradimensional effective field theories need not arise in string theory; however, they can, and when they do, they provide a definite technical framework for calculating the shift in effective parameters - including particle and Planck scales - when branes form and mediate spontaneous dimensional reduction of the universe. This technical framework is completely prescriptive: it offers neither need nor permission for ad hoc assumption, but enables instead a complete and consistent derivation of consequences of extradimensional warp, for both matter and gravity. Gravity, we've seen, can have its effective Planck scale shifted markedly from the fundamental string scale, due to extradimensional warp. Extradimensional field theory for matter lets us probe how the same extradimensional warp affects the effective particle scale: whether it too necessarily shifts, or remains - as previously assumed ad hoc — at the fundamental string scale.

To explore this possible transmutation of the particle scale, I begin with a fully extradimensional field theory with negative bulk cosmological constant, in which both gravity and matter are inherently bulk fields with unified mass scale. The fundamental - extradimensional - particle scale arises from bulk electroweak symmetry-breaking, and coincides with the fundamental - extradimensional - Planck scale. Brane formation establishes a warped background metric, which in turn induces integrated 4D Kaluza Klein effective field theories for both matter and gravity. Note that I take all matter fields to be bulk fields; all physics flows from extradimensional to 4D, controlled by the brane-warped background metric. I calculate the induced 4D effective field theories for both gravity and matter; establish their sensibility; and derive the effective 4D particle and Planck scales they imply, to rigorously validate or refute hierarchy solution. 


\section{Results: Valid 4D effective field theories with hierarchy}

In recent work I apply this extradimensional field theoretic approach to construct viable warped braneworlds, with hierarchy, in three contexts: first, for generic warped extradimensional models of arbitrary codimension [57]; second, for specific example extradimensional defect models of codimension two [43] and three [58]; and third, for the warped geometry of the Randall-Sundrum form [56]. In each, I find the central motivation of warped extra dimension, hierarchy generation, rigorously validated; however, the well-defined 4D effective field theories obtained are interpretable only as either 4D aggregate theories, which encode effects of unresolvably small bulk dimension; or potentially, as localized 4D theories on a single brane. The formalism supports only these interpretations - not the Randall-Sundrum lore of a universal bulk effective theory valid, and differentially resolvable, at each point in the bulk.

In [57] I examine generic warped extradimensional models, of diverse warp and codimension, with bulk electroweak symmetry-breaking from a constant Higgs condensate throughout the bulk. I calculate integrated 4D effective theories for both matter and gravity in the brane-warped background explicitly: deriving for gravity, and for scalars, fermions and gauge fields, their 4D Kaluza-Klein reductions. I find generic results: the effective 4D graviton is always a constant zero mode solution, whose normalization always fixes the induced 4D Planck mass. The effective 4D photon is always a constant gauge zero mode, which always couples universally to electromagnetically charged fields. 4D electromagnetic charge universality thus follows automatically from warped extra dimension, in any model with appropriate bulk electroweak symmetry-breaking. Localization profiles always differ for massless gravitons, photons, massive gauge bosons and massive fermions. This clarifies the structural challenge in constructing warped spacetimes where gravity fully colocalizes entire 4D effective theories on a single brane. However, integrated 4D effective theory still holds for small warped extra dimension, as an unresolved, aggregate theory. Hierarchy solution remains model-dependent, but structurally favored; indeed, the structure of warped extra dimension offers a blueprint for hierarchy generation. For Planck and particle scales are set by distinct physical mechanisms: the Planck scale, by normalization of the graviton zero mode; the particle scale, by lowest Kaluza-Klein masses for Higgs and weak gauge fields. Divergence of these distinct flows occurs readily, inducing hierarchy. Moreover, imposing hierarchy solution typically fixes the allowed extradimensional radius, determining whether a particular model supports the small warped extra dimension scenario.

More concretely, for the most general spherically symmetric warped metric in $D$ dimensions, with $\Lambda_{D}<0$,

$$
d s^{2}=B(r) \bar{g}_{\mu v} d x^{\mu} d x^{v}+A(r) d r^{2}+r^{2} d \Omega^{2},
$$

we may have a flat braneworld solution $\bar{g}_{\mu v}=\eta_{\mu v}, \Lambda_{4}=0$, with background warp factors $A(r)$, $B(r)$. In extradimensional defect models, these warp factors $A(r), B(r)$ are fixed by an uncharged extradimensional defect condensate, with defect core at $r=0$.

Graviton fluctuations $\bar{g}_{\mu v}=\eta_{\mu v}+\bar{h}_{\mu v}$ have modulating bulk wave functions $\bar{h}_{n}(r)$ which obey (in the $l=0$ case)

$$
\frac{1}{B A^{1 / 2} r^{D-5}} \frac{d}{d r}\left(B^{2} A^{-1 / 2} r^{D-5} \frac{d \bar{h}_{n}}{d r}\right)=-m_{n}^{2} \bar{h}_{n}
$$


This is a Sturm-Liouville equation for the graviton mode $\bar{h}_{n}$, with weight $\rho=B A^{1 / 2} r^{D-5}$ and 4D effective Kaluza-Klein mass $m_{n}$. The lowest eigenvalue $m_{n}=0$, a graviton zero mode whose regular solution is simply the constant $\bar{h}_{o}=1 / \sqrt{N}$, mediating a $4 \mathrm{D}$ effective $1 / r^{2}$ gravitational interaction. This mode has spatial probability distribution

$$
\rho\left|\bar{h}_{o}\right|^{2} \sim B A^{1 / 2} r^{D-5} .
$$

Thus the warped metric solution for $A(r)$ and $B(r)$ directly dictates the graviton zero mode's spatial profile: whether, where, and how sharply it localizes in the bulk. The normalization factor $N$ determines the 4D effective Planck mass:

$$
M_{\mathrm{Pl}, 4}^{2}=N M_{\mathrm{Pl}, \mathrm{D}}^{D-2}
$$

Scalars and fermions with bulk electroweak mass $m_{D}$ have modulating bulk Kaluza Klein wave functions $H_{n}(r)$ which obey (again in the $l=0$ case)

$$
\frac{1}{B A^{1 / 2} r^{D-5}} \frac{d}{d r}\left(B^{2} A^{-1 / 2} r^{D-5} \frac{d H_{n}}{d r}\right)-B m_{D}^{2} H_{n}=-m_{n}^{2} H_{n}
$$

These have 4D Kaluza Klein masses $m_{n}$ and Sturm-Liouville weight, as in the gravitational case, $\rho=B A^{1 / 2} r^{D-5}$. The lowest Kaluza Klein mass $m_{1}>0$ fixes the $4 \mathrm{D}$ effective particle scale, with spatial probability distribution

$$
\rho\left|H_{1}\right|^{2} \sim B A^{1 / 2} r^{D-5}\left|H_{1}\right|^{2}
$$

This differs from the massless graviton's spatial profile by the nontrivial eigenfunction $\left|H_{1}\right|^{2}$, and so can be localized differently within the bulk.

Gauge bosons with bulk electroweak mass $m_{a, D}$ have modulating bulk Kaluza Klein wave functions $\mathcal{A}_{\text {an }}(r)$ which obey (again in the $l=0$ case)

$$
\frac{1}{A^{1 / 2} r^{D-5}} \frac{d}{d r}\left(B A^{-1 / 2} r^{D-5} \frac{d \mathcal{A}_{a n}}{d r}\right)-B m_{a, D}^{2} \mathcal{A}_{a n}=-m_{a n}^{2} \mathcal{A}_{a n}
$$

given the transverse gauge choice

$$
0=\sum_{\substack{\text { transverse } \\ X}} \nabla_{X}\left(B^{-1} A_{a}^{X}\right)
$$

which exhausts gauge freedom in the extra (transverse) dimensions only. These have 4D Kaluza Klein masses $m_{a n}$ and Sturm-Liouville weight $\rho_{\mathcal{A}}=A^{1 / 2} r^{D-5}=B^{-1} \rho_{H}$ where $\rho_{H}$ gives the weight for gravitons, scalars, and fermions.

For the photon, with $m_{a, D}=0$, there is again a single regular massless mode, the constant $\mathcal{A}_{a o}=1 / \sqrt{N_{\mathcal{A}}}$. This gauge zero mode becomes the effective 4D photon, with spatial profile

$$
\rho_{\mathcal{A}}\left|\mathcal{A}_{a o}\right|^{2} \sim A^{1 / 2} r^{D-5}
$$

distinct from the massless graviton's profile $B A^{1 / 2} r^{D-5}$. Photons and $1 / r^{2}$ gravity thus generically have distinct localization profiles in the bulk, profiles modulated by the nontrivial warp factor $B(r)$. 
While this might conceivably colocalize photons and 4D gravity on a single brane, in a specific warped model, the generic expectation must be noncoincident distribution - making a single 4D integrated effective theory for matter and gravity valid only as an aggregate effective theory, for unresolvably small warped extra dimension.

For the massive weak bosons, the lowest Kaluza Klein mass $m_{a 1}>0$ fixes the $4 \mathrm{D}$ effective gauge masses, with spatial profile

$$
\rho_{\mathcal{A}}\left|\mathcal{A}_{a 1}\right|^{2} \sim A^{1 / 2} r^{D-5}\left|\mathcal{A}_{a 1}\right|^{2}
$$

This differs not only from graviton, scalar, and fermion spatial profiles, but also from the photon's spatial profile, by the nontrivial eigenfunction $\left|\mathcal{A}_{a 1}\right|^{2}$. Thus we have generic and rampant diversity in which the 4D effective particles from four classes - massless bulk gravitons, scalars and fermions; massive bulk scalars and fermions; bulk photons; and massive bulk weak gauge bosons — each localize differently within the bulk.

We see here the structural predisposition to hierarchy generation — due to the distinct physical mechanisms driving the $4 \mathrm{D}$ effective Planck mass to $M_{\mathrm{Pl}, 4}^{2}=N M_{\mathrm{Pl}, \mathrm{D}}^{D-2}$, and the $4 \mathrm{D}$ effective particle masses to $m_{1}, m_{a 1}$ for the Higgs and weak gauge bosons. Detailed examination of this divergence, however, remains model-dependent, as specific warped solutions $A(r), B(r)$ fix both $N$ and the Kaluza Klein masses $m_{1}, m_{a 1}: N$ quite directly, but $m_{1}$ and $m_{a 1}$ in roundabout, complex ways.

With Domokos [58] and with Toebbe [43], I establish numerical solutions for two specific warped extradimensional defect models, with 4D induced matter effective theories, localized gravity, and hierarchy solution all rigorously validated. These involve, in the first case, 4D matter effective theory in the Benson-Cho extradimensional global monopole model [48]; in the second, a new warped solution and 4D effective theory for matter and gravity, for an extradimensional global string. In both cases, hierarchy solution remains robust, as Planck and particle scales diverge hierarchically. For both, the Planck scale grows hierarchically, while the particle scale shifts little: in the monopole case, flowing to or remaining near a natural value of order $\left|\Lambda_{7}\right|$. Thus both are characterized by unified extradimensional matter and gravity at the TeV scale, inducing 4D Planck scale gravity and 4D TeV scale matter. Both involve diversely localized fields, with 4D integrated effective theories valid only for extradimensional radii too small to resolve; yet both numerically accommodate this possibility.

In [56], I apply this same extradimensional field theoretic approach to the Randall-Sundrum warped background. I view both matter and gravity as inherently $5 \mathrm{D}$, as bulk fields with unified mass scale - with 5D particle scale again set by bulk electroweak symmetry-breaking. Here, however, technicalities arise, as $Z_{2}$-orbifold symmetry requires bulk fermion masses to be $Z_{2}$-odd in the bulk, assuming a kink profile $m \operatorname{sign}(y)$. This is accomplished by a $Z_{2}$-odd Higgs vev, with comparable kink profile, yielding uniform masses for gauge and Higgs particles. These yield integrated Kaluza-Klein effective theories for matter which have appeared in the literature before (for gravity, in [7]; for bulk scalars, in [59]; for bulk gauge fields, in [21]; and for bulk fermions, in [60, 61, 62]). In my paradigm, though, these effective matter fields are again the only matter fields; all fields are bulk fields, and all physics flows from the bulk to 4D.

This unified extradimensional approach rigorously validates hierarchy solution, in a more constrained interpretation than Randall-Sundrum: unified 5D matter and gravity at the Planck scale induces 4D Planck scale gravity and 4D TeV scale matter, when an extra dimension of size $12 M_{\mathrm{Pl}}^{-1}$ 
warps due to brane formation. Our formalism sheds the ambiguity latent in Randall-Sundrum: the unified bulk scale must lie at the Planck mass, as the effective 4D Planck mass changes little, while the effective 4D particle mass-scale becomes hierarchically suppressed. Note that this instantiates a distinct avenue to warp-generated hierarchy, unlike the extradimensional defect models above, where unified bulk scale at a $\mathrm{TeV}$ induces a little-changed 4D particle scale and a hierarchically enhanced 4D Planck scale. In each case, a hierarchical divergence arises between induced 4D particle and Planck scales; yet whether that divergence arises from enhancement of the Planck scale (as in the extradimensional defect models) or a suppression of the particle scale (as for the Randall-Sundrum background) remains model-dependent.

For the Randall-Sundrum background, this extradimensional field theoretic approach automatically solves a second problem: the dimensionally reduced matter effective field theory preserves both electromagnetic and weak charge universality. This offers a rare achievement: a field theoretic dimensional reduction with demonstrable weak charge universality. Moreover, while fields localize differentially in the bulk - massless gravitons on the Planck brane; massive matter fields on the particle brane; photons smeared throughout the bulk - the integrated 4D effective field theory remains sensible for all fields. This is because hierarchy demands such a small warped extra dimension $-12 M_{\mathrm{Pl}}^{-1}-$ that varied localization in the extra dimension remains unresolved. The observationally valid 4D effective theory is the smeared, aggregate one obtained by integration. Thus we have in the Randall-Sundrum case a viable warped model, one which: 1) induces a single dimensionally reduced 4D theory of clear validity for both matter and gravity; and 2) generates hierarchy, while preserving both electromagnetic and weak charge universality. Yet this viable theory describes not the large and resolvable bulk interpreted by Randall-Sundrum, but an aggregate 4D theory due to a truly tiny — but warped — extra dimension.

\section{Conclusions}

I introduced the warped extradimensional paradigm, then critiqued its shortcomings: overdominance of a single toy model (Randall-Sundrum); asymmetric treatment of matter and gravity 4D effective field theories; and eclectic spatial interpretation of integrated effective field theories.

I then defined a unified extradimensional theory for matter and gravity, in a generic warped extradimensional background with bulk electroweak symmetry-breaking. I examined Kaluza-Klein reduction in this warped background, deriving integrated 4D effective field theories for gravity, and for free scalars, fermions, and gauge fields. I discussed induced 4D Planck and particle scales, and localization of diverse modes. I noted that massless modes have universal form, determining distinct localization profiles for gravitons and photons. This generically obstructs construction of warped spacetimes with fully colocalized local 4D effective theories. Hierarchy solution allows an alternative: smeared 4D effective theories, from unresolvably small extra dimension. Because the divergence between induced particle and Planck scales typically scales with extradimensional radius, hierarchy solution fixes, for each model, a scenario of definite large or small extra dimension. In either case - whether colocalized or smeared over unresolvably small extra dimension whenever the induced 4D effective field theories are sensible, they generically generate hierarchy and preserve electromagnetic charge universality. 
I described specific warped extradimensional defect models, which induce valid 4D effective field theories with unresolvably small extra dimension and rigorously validated hierarchy solution. In them a unified bulk scale at a $\mathrm{TeV}$ induces $4 \mathrm{D} \mathrm{TeV}$ scale matter and Planck scale gravity. In the Randall-Sundrum background, instead, unified 5D matter and gravity originates at the Planck scale, inducing 4D Planck scale gravity and 4D TeV scale matter when an extra dimension of unresolvably tiny size $12 M_{\mathrm{Pl}}^{-1}$ warps. This case, as well, yields well-defined 4D effective field theories, with rigorously validated hierarchy solution, electromagnetic and weak charge universality.

\section{References}

[1] O. Klein, Quantum Theory And Five-Dimensional Theory Of Relativity, Z. Phys. 37, 895 (1926) [Surveys High Energ. Phys. 5, 241 (1986)]; reprinted in English translation in Modern Kaluza-Klein Theories, T. . Appelquist, A. . Chodos and P. G. Freund (Addison-Wesley, Menlo Park, 1987).

[2] T. Kaluza, On The Problem Of Unity In Physics, Sitzungsber. Preuss. Akad. Wiss. Berlin (Math. Phys. ) 1921, 966 (1921); reprinted in English translation in Modern Kaluza-Klein Theories, ed. T. . Appelquist, A. . Chodos and P. G. Freund (Addison-Wesley, Menlo Park, 1987).

[3] For introduction to branes in string theory, see J. Polchinski, Dirichlet-Branes and Ramond-Ramond Charges, Phys. Rev. Lett. 75, 4724 (1995) [hep-th/9510017]; J. Polchinski, Lectures on D-branes, in Boulder 1996, Fields, strings and duality, ed. C. Efthimiou, B. Greene, J. Efthimiou (World Scientific, Singapore, 1997) 293-356 [hep-th/9611050].

[4] For an overview of branes in supergravity and M theory, see M. J. Duff, Lectures on branes, black holes and anti-de Sitter space, in Strings, Branes and Gravity: Lecture Notes TASI 99, ed. J. Harvey and S. Kachru (World Scientific, Singapore, 2000) [hep-th/9912164].

[5] N. Arkani-Hamed, S. Dimopoulos and G. Dvali, The hierarchy problem and new dimensions at a millimeter, Phys. Lett. B429, 263 (1998) [hep-ph/9803315]; N. Arkani-Hamed, S. Dimopoulos and G. Dvali, Phenomenology, astrophysics and cosmology of theories with sub-millimeter dimensions and TeV scale quantum gravity, Phys. Rev. D59, 086004 (1999) [hep-ph/9807344].

[6] L. Randall and R. Sundrum, An alternative to compactification, Phys. Rev. Lett. 83, 4690 (1999) [hep-th/9906064].

[7] L. Randall and R. Sundrum, A large mass hierarchy from a small extra dimension, Phys. Rev. Lett. 83, 3370 (1999) [hep-ph/9905221].

[8] P. Binetruy, C. Deffayet and D. Langlois, Non-conventional cosmology from a brane-universe, Nucl. Phys. B 565, 269 (2000) [hep-th/9905012]; P. Binetruy, C. Deffayet, U. Ellwanger and D. Langlois, Brane cosmological evolution in a bulk with cosmological constant, Phys. Lett. B 477, 285 (2000) [hep-th/9910219];

[9] C. Csaki, M. Graesser, C. F. Kolda and J. Terning, Cosmology of one extra dimension with localized gravity, Phys. Lett. B 462, 34 (1999) [hep-ph/9906513].

[10] J. M. Cline, C. Grojean and G. Servant, Cosmological expansion in the presence of extra dimensions, Phys. Rev. Lett. 83, 4245 (1999) [hep-ph/9906523].

[11] E. E. Flanagan, S. H. Tye and I. Wasserman, Cosmological expansion in the Randall-Sundrum brane world scenario, Phys. Rev. D 62, 044039 (2000) [hep-ph/9910498].

[12] A. Lukas, B. A. Ovrut and D. Waldram, Boundary inflation, Phys. Rev. D 61, 023506 (2000) [hep-th/9902071]. 
[13] J. M. Cline and J. Vinet, Order rho**2 corrections to Randall-Sundrum I cosmology, JHEP 0202, 042 (2002) [hep-th/0201041].

[14] P. Brax and A. C. Davis, Cosmological evolution on self-tuned branes and the cosmological constant,JHEP 0105, 007 (2001) [hep-th/0104023]; P. Brax and A. C. Davis, Cosmological solutions of supergravity in singular spaces, Phys. Lett. B 497, 289 (2001) [hep-th/0011045].

[15] R. Maartens, D. Wands, B. A. Bassett and I. Heard, Chaotic inflation on the brane, Phys. Rev. D 62, 041301 (2000) [hep-ph/9912464].

[16] E. J. Copeland, A. R. Liddle and J. E. Lidsey, Steep inflation: Ending braneworld inflation by gravitational particle production, Phys. Rev. D 64, 023509 (2001) [astro-ph/0006421].

[17] Y. Himemoto and M. Sasaki, Brane-world inflation without inflaton on the brane, Phys. Rev. D 63, 044015 (2001) [gr-qc/0010035]; Y. Himemoto, T. Tanaka and M. Sasaki, A bulk scalar in the braneworld can mimic the 4d inflaton dynamics, Phys. Rev. D 65, 104020 (2002) [gr-qc/0112027].

[18] This is nicely reviewed in D. Langlois, Brane cosmology: An introduction, Prog. Theor. Phys. Suppl. 148, 181 (2003) [hep-th/0209261].

[19] G. R. Dvali, G. Gabadadze and M. Porrati, 4D gravity on a brane in 5D Minkowski space, Phys. Lett. B 485, 208 (2000) [hep-th/0005016]; see review in G. Gabadadze, ICTP lectures on large extra dimensions, hep-ph/0308112.

[20] W. D. Goldberger and M. B. Wise, Modulus stabilization with bulk fields, Phys. Rev. Lett. 83, 4922 (1999) [hep-ph/9907447].

[21] A. Pomarol, Gauge bosons in a five-dimensional theory with localized gravity, Phys. Lett. B 486, 153 (2000) [hep-ph/9911294].

[22] See nice review in M. Carena, E. Ponton, T. M. Tait and C. E. Wagner, Opaque branes in warped backgrounds, Phys. Rev. D 67, 096006 (2003) [hep-ph/0212307].

[23] P. Horava and E. Witten, Heterotic and type I string dynamics from eleven dimensions, Nucl. Phys. B 460, 506 (1996) [hep-th/9510209];

[24] S. Kachru, R. Kallosh, A. Linde and S. P. Trivedi, De Sitter vacua in string theory, Phys. Rev. D 68, 046005 (2003) [hep-th/0301240].

[25] L. Susskind, The anthropic landscape of string theory, [hep-th/0302219].

[26] M. R. Douglas, The statistics of string / M theory vacua, JHEP 0305, 046 (2003) [hep-th/0303194].

[27] S. Ashok and M. R. Douglas, Counting flux vacua, JHEP 0401, 060 (2004) [hep-th/0307049].

[28] T. Banks, M. Dine and E. Gorbatov, Is there a string theory landscape?, JHEP 0408, 058 (2004) [hep-th/0309170].

[29] F. Denef and M. R. Douglas, Distributions of flux vacua, JHEP 0405, 072 (2004) [hep-th/0404116].

[30] R. H. Brandenberger and C. Vafa, Superstrings In The Early Universe, Nucl. Phys. B 316, 391 (1989).

[31] S. Alexander, R. H. Brandenberger and D. Easson, Brane gases in the early universe, Phys. Rev. D 62, 103509 (2000) [hep-th/0005212].

[32] R. Easther, B. R. Greene and M. G. Jackson, Cosmological string gas on orbifolds, Phys. Rev. D 66, 023502 (2002) [hep-th/0204099].

[33] A. G. Cohen and D. B. Kaplan, Solving the hierarchy problem with noncompact extra dimensions, Phys. Lett. B470, 52 (1999) [hep-th/9910132]. 
[34] A. Chodos and E. Poppitz, Warp factors and extended sources in two transverse dimensions, Phys. Lett. B 471, 119 (1999) [hep-th/9909199].

[35] I. Olasagasti and A. Vilenkin, Gravity of higher-dimensional global defects, Phys. Rev. D 62, 044014 (2000) [hep-th/0003300].

[36] R. Gregory, Nonsingular global string compactifications, Phys. Rev. Lett. 84, 2564 (2000) [hep-th/9911015].

[37] T. Gherghetta and M. E. Shaposhnikov, Localizing gravity on a string-like defect in six dimensions, Phys. Rev. Lett. 85, 240 (2000) [hep-th/0004014].

[38] E. Ponton and E. Poppitz, Gravity localization on string-like defects in codimension two and the AdS/CFT correspondence, JHEP 0102, 042 (2001) [hep-th/0012033].

[39] M. Giovannini, H. Meyer and M. E. Shaposhnikov, Warped compactification on Abelian vortex in six dimensions, Nucl. Phys. B 619, 615 (2001) [hep-th/0104118].

[40] R. Gregory and C. Santos, Spacetime structure of the global vortex, Class. Quant. Grav. 20, 21 (2003) [hep-th/0208037].

[41] B. de Carlos and J. M. Moreno, A cigar-like universe, JHEP 0311, 040 (2003) [hep-th/0309259].

[42] B. de Carlos and J. M. Moreno, Regular compactifications and Higgs model vortices, hep-th/0405144.

[43] K. Benson and J. Toebbe, Braneworld physics in a warped global string background, in preparation.

[44] O. DeWolfe, D. Z. Freedman, S. S. Gubser and A. Karch, Modeling the fifth dimension with scalars and gravity, Phys. Rev. D 62, 046008 (2000) [hep-th/9909134].

[45] T. Gherghetta, E. Roessl and M. E. Shaposhnikov, Living inside a hedgehog: Higher-dimensional solutions that localize gravity, Phys. Lett. B 491, 353 (2000) [hep-th/0006251].

[46] S. Randjbar-Daemi and M. E. Shaposhnikov, On some new warped brane world solutions in higher dimensions, Phys. Lett. B 491, 329 (2000) [hep-th/0008087].

[47] S. H. Moon, S. J. Rey and Y. B. Kim, Global p-brane world: A new approach to stable mass hierarchy, Nucl. Phys. B 602, 467 (2001) [hep-th/0012165].

[48] K. Benson and I. Cho, A universe in a global monopole, Phys. Rev. D64, 065026 (2001) [hep-th/0104067].

[49] J. Vinet and J. M. Cline, Can codimension-two branes solve the cosmological constant problem?, hep-th/0406141.

[50] V. A. Rubakov and M. E. Shaposhnikov, Do We Live Inside A Domain Wall?, Phys. Lett. B 125, 136 (1983).

[51] V. A. Rubakov and M. E. Shaposhnikov, Extra Space-Time Dimensions: Towards A Solution To The Cosmological Constant Problem, Phys. Lett. B 125, 139 (1983).

[52] G. R. Dvali and M. A. Shifman, Domain walls in strongly coupled theories, Phys. Lett. B 396, 64 (1997) [Erratum-ibid. B 407, 452 (1997)] [hep-th/9612128].

[53] V. A. Rubakov, Large and infinite extra dimensions: An introduction, Phys. Usp. 44, 871 (2001) [Usp. Fiz. Nauk 171, 913 (2001)] [hep-ph/0104152].

[54] I. Oda, Localization of matters on a string-like defect, Phys. Lett. B 496, 113 (2000) [hep-th/0006203]. 
[55] S. Randjbar-Daemi and M. E. Shaposhnikov, Fermion zero-modes on brane-worlds, Phys. Lett. B 492, 361 (2000) [hep-th/0008079].

[56] K. Benson, On hierarchy, charge universality, and 4D effective theory in Randall-Sundrum models, hep-th/0407276.

[57] K. Benson, Matter and gravity in warped extradimensional models, in preparation.

[58] K. Benson and S. Domokos, Hierarchy and 4D effective theory from a warped global monopole, in preparation.

[59] W. D. Goldberger and M. B. Wise, Bulk fields in the Randall-Sundrum compactification scenario, Phys. Rev. D 60, 107505 (1999) [hep-ph/9907218].

[60] T. Gherghetta and A. Pomarol, Bulk fields and supersymmetry in a slice of AdS, Nucl. Phys. B 586, 141 (2000) [hep-ph/0003129].

[61] Y. Grossman and M. Neubert, Neutrino masses and mixings in non-factorizable geometry, Phys. Lett. B 474, 361 (2000) [hep-ph/9912408].

[62] S. Chang, J. Hisano, H. Nakano, N. Okada and M. Yamaguchi, Bulk standard model in the Randall-Sundrum background, Phys. Rev. D 62, 084025 (2000) [hep-ph/9912498]. 